\title{
Profile of rheumatology patients willing to report adverse drug reactions: bias from selective reporting
}

\author{
Dragana Protićl \\ Nada Vujasinović-Stupar ${ }^{2}$ \\ Zoran Bukumirić ${ }^{3}$ \\ Slavica Pavlov-Dolijanović ${ }^{4}$ \\ Snežana Baltić ${ }^{5}$ \\ Slavica Mutavdžin ${ }^{6}$ \\ Ljiljana Marković-Denić ${ }^{7}$ \\ Marija Zdravković ${ }^{8}$ \\ Zoran Todorović' \\ 'Department of Pharmacology, Clinical \\ Pharmacology and Toxicology, Faculty \\ of Medicine, University of Belgrade, \\ Belgrade, Serbia; ${ }^{2}$ Department 2, \\ Institute of Rheumatology, Faculty \\ of Medicine, University of Belgrade, \\ Belgrade, Serbia; ${ }^{3}$ Institute for Medical \\ Statistics and Informatics, Faculty \\ of Medicine, University of Belgrade, \\ Belgrade, Serbia; ${ }^{4}$ Department 5 , \\ Institute of Rheumatology, Faculty \\ of Medicine, University of Belgrade, \\ Belgrade, Serbia; ${ }^{5}$ Department 5 , \\ Institute of Rheumatology, Belgrade, \\ Serbia; 'Institute of Physiology \\ "Rihard Burjan", Faculty of Medicine, \\ University of Belgrade, Belgrade, \\ Serbia; ${ }^{7}$ Institute of Epidemiology, \\ Faculty of Medicine, University \\ of Belgrade, Belgrade, Serbia; \\ ${ }^{8}$ Department of Cardiology, Medical \\ Center "Bežanijska kosa”, Faculty \\ of Medicine, University of Belgrade, \\ Belgrade, Serbia
}

Correspondence: Zoran Todorović Department of Pharmacology,

Clinical Pharmacology and Toxicology, Faculty of Medicine, University of Belgrade, Dr Subotića starijeg I, PO Box 38, Belgrade II I29, Serbia

Tel +38 I II 3643389

Fax +38I I| 3643397

Email zoran.todorovic@mfub.bg.ac.rs
This article was published in the following Dove Press journal:

Patient Preference and Adherence

2 February 2016

Number of times this article has been viewed

Background: Adverse drug reactions (ADRs) have a significant impact on human health and health care costs. The aims of our study were to determine the profile of rheumatology patients willing to report ADRs and to identify bias in such a reporting system.

Methods: Semi-intensive ADRs reporting system was used in our study. Patients willing to participate $(\mathrm{N}=261)$ completed the questionnaire designed for the purpose of the study at the hospital admission. They were subsequently classified into two groups according to their ability to identify whether they had experienced ADRs during the previous month. Group 1 included 214 out of 261 patients who were able to identify ADRs, and group 2 consisted of 43 out of 261 patients who were not able to identify ADRs in their recent medical history.

Results: Group 1 patients were more significantly aware of their diagnosis than the patients from group 2. Marginal significance was found between rheumatology patients with and without neurological comorbidities regarding their awareness of ADRs. The majority of patients reported ADRs of cytotoxic drugs. The most reported ADRs were moderate gastrointestinal discomforts.

Conclusion: We may draw a profile of rheumatological patients willing to report ADRs: 1) The majority of them suffer from systemic inflammatory diseases and are slightly more prone to neurological comorbidities. 2) They are predominantly aware of their diagnosis but less able to identify the drugs that may cause their ADRs. 3) They tend to report mainly moderate gastrointestinal ADRs; that is, other cohorts of patients and other types of ADRs remain mainly undetected in such a reporting, which could represent a bias. Counseling and education of patients as well as developing a network for online communication might improve patients' reporting of potential ADRs.

Keywords: adverse drug reactions, patient reporting system, rheumatology, bias

\section{Introduction}

According to Meyboom et al, adverse drug reaction (ADR) could be defined as "a response to a drug which is noxious and unintended", while a broader term side effect could be defined as "any unintended effect of a pharmaceutical product occurring at doses normally used in humans, which is related to the pharmacological properties of the drug"; that is, side effect involves both therapeutic and adverse effects which are not intended. ${ }^{1}$ ADRs to prescribed medicines significantly affect human health and increase health care costs. Incidence of serious ADRs was 6.7\% (with fatal outcome in $0.32 \%$ ) of hospitalized patients in hospitals in the USA. Therefore, ADRs are among leading causes of death in the USA. ${ }^{2}$ In addition, $6.5 \%$ of admissions to UK hospitals were due to ADRs; three quarters of those were preventable ADRs, and $>2 \%$ of them caused death. ${ }^{3}$ Patient reporting of potential ADRs has an important role in 
pharmacovigilance as a valuable source of information. ${ }^{4,5}$ Pharmacovigilance systems in many countries (eg, the UK, the USA, Canada, Australia, New Zealand, and Sweden) have incorporated patient's reports. According to the current national regulations, patients in Serbia are permitted to report ADRs. ${ }^{6,7}$ Serbian Medicines and Medical Devices Agency (ALIMS) has established such a reporting system. Patient's spontaneous reports are submitted directly to the ALIMS (http://www.alims.gov.rs/eng/pharmacovigilance/). According to the 2013 Annual Report, nine out of 1,159 adverse events $(<1 \%)$ were directly submitted to the ALIMS by patients.

The aims of our research were 1) to determine patients' ability to identify and rate ADRs according to their own experience, 2) to assess possible bias in such a reporting system (ie, which ADR patients were more prone to report), and 3) to detect associated factors which facilitate identification of ADRs by patients. Finally, these pieces of information will allow us to identify potential reporting bias (ie, to draw the profile of a patient willing to report ADRs and characteristics of the data that will be gathered in such a way), and to overcome such a bias.

\section{Methods}

\section{Study site}

We studied patient's reporting of ADRs at the Institute of Rheumatology in Belgrade, Serbia, Clinical Department 2. The study period lasted from September 1, 2012 to September 1, 2013. Belgrade's Institute of Rheumatology affiliates with an academic medical center with full- and part-time teacher clinicians.

\section{Study subjects}

Inclusion criteria were as follows: a) age $>18$ years, b) diagnosis of chronic rheumatic disease, and c) stable antirheumatic therapy. On the other hand, the following participants were excluded: patients who disagreed to participate/ sign the consent, b) patients with severely debilitated health states, c) mentally incapacitated patients, and d) patients with language barriers.

\section{Study protocol}

Semi-intensive ADRs reporting system was used in our survey. The study has been approved by the Ethics Committee of the Institute of Rheumatology, Belgrade (EtiČki odbor, Institut za reumatologiju, Beograd). In particular, the Ethics Committee specifically approved the method of consent used in our study.
After the admission to the Institute, each patient was provided with a copy of the questionnaire designed for the purpose of this study. ${ }^{8}$ (The questionnaire can be made available to researchers on request.) The questionnaire was made according to the suggestions of Edwards ${ }^{9}$ and Somers et al. ${ }^{10}$ The questionnaire introduction contained an explanation that data would be used solely for the assessment of drug safety. If they agreed to participate (written agreement), patients completed the questionnaire and submitted it to the nurse in the ward. The questionnaire consists of five sections, with questions about the following: 1) general patient characteristics, duration/timeline, and information about his/her illness (rheumatic diseases and whether the patient had any other diseases); 2) history of drug use during the previous month (questions on the therapeutic groups and compliance/adherence); 3) ADRs experienced during the previous month using structured checklists; 4) subjective patient's assessment of the severity of ADRs experienced during the previous month using a 5-point visual analog scale; and 5) patient's opinion on other possible causes of ADRs experienced during the previous month. The questionnaire has been designed for the purpose of the study and adjusted to standard existing questionnaires for patients' spontaneous reporting of ADRs. Content/face validity of the questionnaire has been assessed through interviews with patients and health professionals from the ward.

\section{Statistical analysis}

The results are presented in the form of descriptive statistics (nominal scale) and median and interquartile range (25th-75th centile) (score values). Statistical analysis was carried out using the chi-square test, Fisher's exact test, or MannWhitney $U$-test, depending on the scale of measurement.

The missing data due to unanswered questions were assumed to be distributed at random, and they were excluded from the analysis. Accordingly, only the available data were analyzed. ${ }^{11}$

The entire statistical analysis was performed by using GraphPad Prism/Instat 1.1 software (GraphPad Inc, San Diego, CA, USA) and SPSS for Windows (Version 16.0, Released 2007; SPSS Inc., Chicago, IL, USA). A $P$-value of $<0.05$ was considered significant $(P>0.05$ is nonsignificant).

\section{Results}

Two hundred and sixty-one patients were included in our study. Two hundred and fifty-seven out of 261 patients (98.5\%) answered the question about their own ability to identify ADRs in personal medical history (response rate 
Table I Demographic characteristics of the study population and awareness of their diagnosis

\begin{tabular}{llll}
\hline Parameter & Group I & Group 2 & P-value \\
\hline $\begin{array}{l}\text { Age, } \bar{x} \pm \text { SD (years) } \\
\text { Sex, } \mathrm{n}(\%)\end{array}$ & $58.0 \pm 10.8$ & $55.8 \pm 12.9$ & 0.297 \\
$\quad$ Male & $9(20.9 \%)$ & $35(16.4 \%)$ & 0.467 \\
$\quad$ Female & $34(79.1 \%)$ & $179(83.6 \%)$ & \\
Employment, $\mathrm{n}(\%)$ & & & \\
$\quad$ Employed & $18(42.9 \%)$ & $93(43.7 \%)$ & 0.992 \\
$\quad$ Unemployed & $8(19.0 \%)$ & $41(19.2 \%)$ & \\
$\quad$ Retired & $16(38.1 \%)$ & $79(37.1 \%)$ & \\
Informed about diagnosis, $\mathrm{n}(\%)$ & & & \\
$\quad$ Informed & $22(52.4 \%)$ & $66(32.0 \%)$ & 0.012 \\
$\quad$ Not informed & $20(47.6 \%)$ & $140(68.0 \%)$ & \\
Duration of rheumatic disease, $\mathrm{n}(\%)$ & & & \\
$\quad<\mathrm{I}$ year & $7(16.3 \%)$ & $62(28.4 \%)$ & 0.098 \\
$\quad>I$ year & $36(83.7 \%)$ & $156(71.6 \%)$ & \\
Region, $\mathrm{n}(\%)$ & & & \\
$\quad$ Vojvodina & $4(9.8 \%)$ & $27(13.2 \%)$ & 0.773 \\
$\quad$ Belgrade & $17(41.5 \%)$ & $88(42.9 \%)$ & \\
$\quad$ Central Serbia & $20(48.8 \%)$ & $90(43.9 \%)$ & \\
\hline
\end{tabular}

Notes: Group I, patients aware of ADRs in their medical history; group 2, patients no aware of ADRs in their medical history. Data in bold indicates statistical significance. Abbreviations: SD, standard deviation; ADRs, adverse drug reactions.

[RR] of $98.5 \%)$. Of those, $214(82 \%)$ were able to identify whether they had experienced ADRs before, and 43 patients (16.5\%) could not do that (groups 1 and 2, respectively).

A hundred and ninety-two patients (73.6\%) included in our study had suffered for $>1$ year from rheumatic diseases. Group 1 patients were more significantly aware of their diagnosis than patients from group $2(P<0.05)$. Groups 1 and 2 were similar with respect to their other characteristics (age, sex, occupation, duration of disease, and place of living) (Table 1).

We have analyzed comorbidities of the rheumatology patients who participated in our study (181/261 or $69.3 \%$ of patients reported comorbidities). There is no significant difference between groups 1 and 2 regarding distribution of the reported comorbidities (not shown in the table). Significantly more rheumatology patients with neurological comorbidities (nervous system disorders) belong to group 1, while such a difference was not found in subgroups with other comorbidities (Table 2).

Among 214 patients who were able to identify whether they had had ADRs, 164 (76.6\%) patients were positive that they had not experienced ADRs before. There is no significant difference between groups of patients with and without ADRs in relation to their age, sex, occupation, information on their diagnosis, duration of disease, and place of living (not shown in the table). On the other hand, among 50 patients who were positive on ADR experience, causative drug was identified in just 18 cases (cytotoxic drugs, 10/18; anti-inflammatory agents, 2/18; other drugs, 6/18).

There is a similar distribution of the diagnosis of musculoskeletal and connective tissue disorders among the patients from groups 1 and 2 (Figure 1). In both groups, patients with systemic connective tissue diseases and inflammatory polyarthropathies most often reported ADRs (Figure 2).

Gastrointestinal disorders predominate over other ADR localizations (Figure 3), while the majority of ADRs reported are moderate on 5-point scale (Figure 4).

\section{Discussion}

Spontaneous patient reporting of suspected ADRs could enhance knowledge about the possible side effects of drugs. ${ }^{12}$ There is an increasing trend toward sharing treatment decision-making roles between patients and physicians. ${ }^{5}$ In addition, there are many patient social networks and forums such as "Ask a Patient" or "iMedx", which collect such data directly from patients or other drug consumers. The researchers believe that such forums are a rich resource of information about ADRs and drug-drug interaction (http://www.meco-project.eu/). Knezevic et al discussed the role of online social networks in pharmacovigilance, and found that patients were less prone to reporting serious or unexpected ADRs. ${ }^{13}$ However, a causal relationship with the drug was strong in patients' reports suggesting high

Table 2 Comorbidities in the population of rheumatology patients studied

\begin{tabular}{llll}
\hline Comorbidities & Group I & Group 2 & P-value \\
\hline Cardiac and vascular disorders, $\mathrm{n}(\%)$ & $12(30.8 \%)$ & $70(34.8 \%)$ & 0.625 \\
Neoplasms, $\mathrm{n}(\%)$ & $0(0.0 \%)$ & $\mathrm{II}(5.5 \%)$ & 0.220 \\
Nervous system disorders, $\mathrm{n}(\%)$ & $0(0.0 \%)$ & $16(8.0 \%)$ & $\mathbf{0 . 0 4 9}$ \\
Renal and urinary disorders, $\mathrm{n}(\%)$ & $\mathrm{I}(2.5 \%)$ & $5(2.5 \%)$ & 1.000 \\
Metabolism and nutrition disorders, $\mathrm{n}(\%)$ & $4(10.0 \%)$ & $36(18.0 \%)$ & 0.215 \\
Gastrointestinal disorders, $\mathrm{n}(\%)$ & $2(5.1 \%)$ & $24(12.0 \%)$ & 0.270 \\
\hline
\end{tabular}

Notes: Group I, patients aware of ADRs in their medical history; group 2, patients not aware of ADRs in their medical history. Data in bold indicates statistical significance.

Abbreviation: ADRs, adverse drug reactions. 


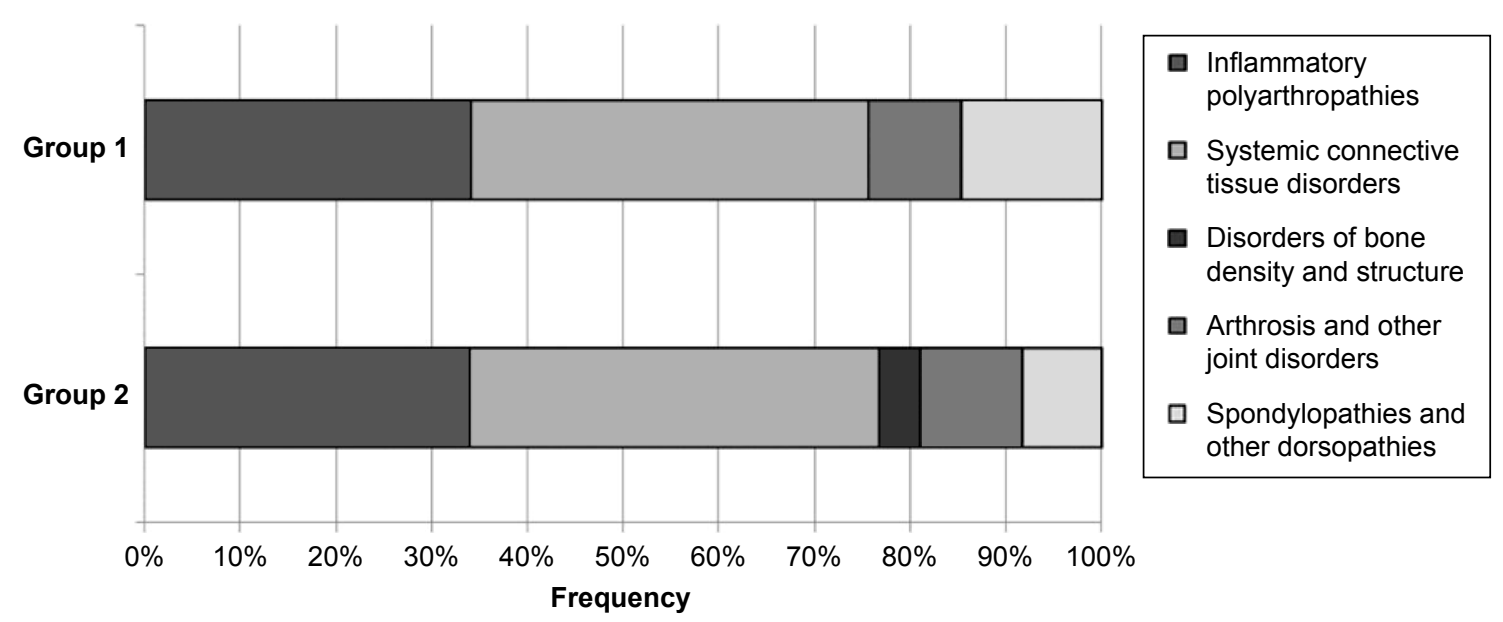

Figure I Diagnoses and awareness of adverse drug reactions in the population of rheumatology patients studied.

Notes: Group I, patients aware of adverse drug reactions in their medical history; group 2, patients not aware of adverse drug reactions in their medical history. Stacked percentage bars represent the distribution of patients from groups $I$ and 2 according to their diagnosis.

sensitivity of this instrument for ADRs reporting. A similar observation has been published by DeWitt and Sorofman who stated that "people have knowledge about ADR symptoms that is substantially accurate". ${ }^{14}$

Patient reporting scheme in pharmacovigilance significantly contributes to the post-marketing of drug safety data collection. There is evidence of if and how patients have been integrated into the pharmacovigilance system. Participants of the conference Health Action International 2005 called for greater sharing of data and experience of patient reporting. It is well known that some countries (eg, Australia, Canada, New Zealand, Norway, and Denmark) have developed a system for patient reporting of ADRs. There are some differences in the way how various countries handle patient reports. ${ }^{15}$ Serbia developed a system for evidence of suspected ADRs by patients 2 years ago. However,

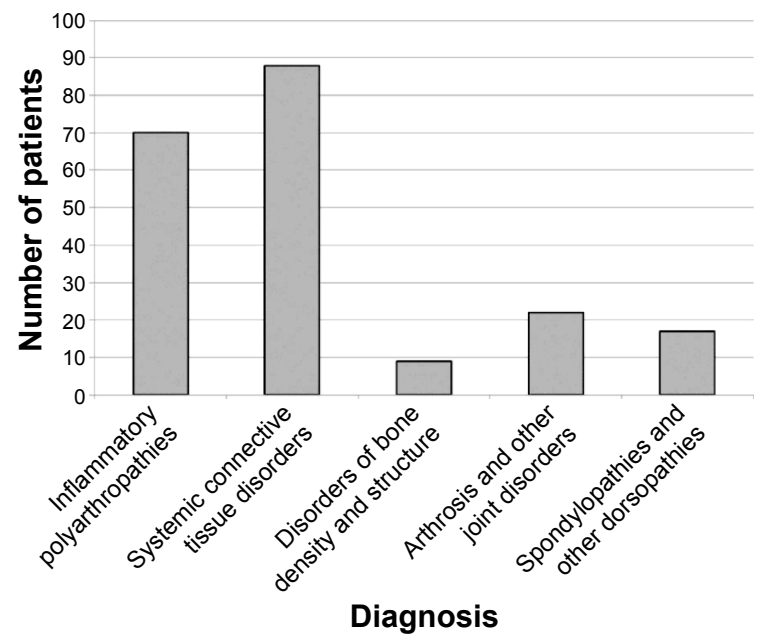

Figure 2 Frequency histogram of adverse drug reactions reported in rheumatology patients with different diagnosis (vertical bars). public awareness of patient reporting scheme is low, and posters or television advertisements about the significance of patients in health care system in Serbia are still lacking. Accordingly, drug customers in Serbia are not aware of the important role they have in pharmacovigilance system. Currently, the data on patients' reports about ADRs are not available in Serbia.

According to the ALIMS records, number of individual ADR reports (cases) in our country steadily increased from 75 in 2004 to 1,173 in 2013. Despite such an increase, the number of ADRs reported annually is still far from the expected goal, that is, $\sim 1,500$ of reports per year. In addition, the report rate of large clinical centers in Serbia is still far from satisfying.

In our study, the RR was $\sim 100 \%$; that is, almost all the patients accepted to participate in the survey, although

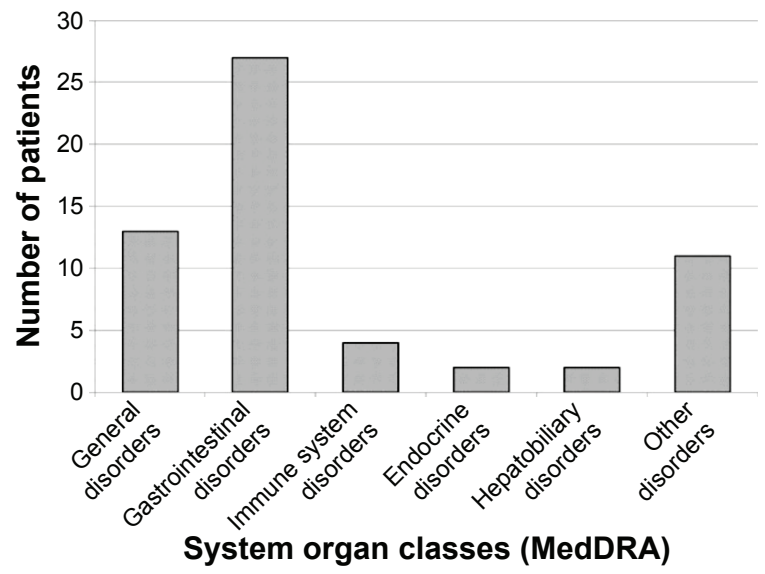

Figure 3 Distribution of adverse drug reactions reported according to the MedDRA system organ classes (frequency histogram).

Abbreviations: ADRs, adverse drug reactions; MedDRA, Medical Dictionary for Regulatory Activities. 


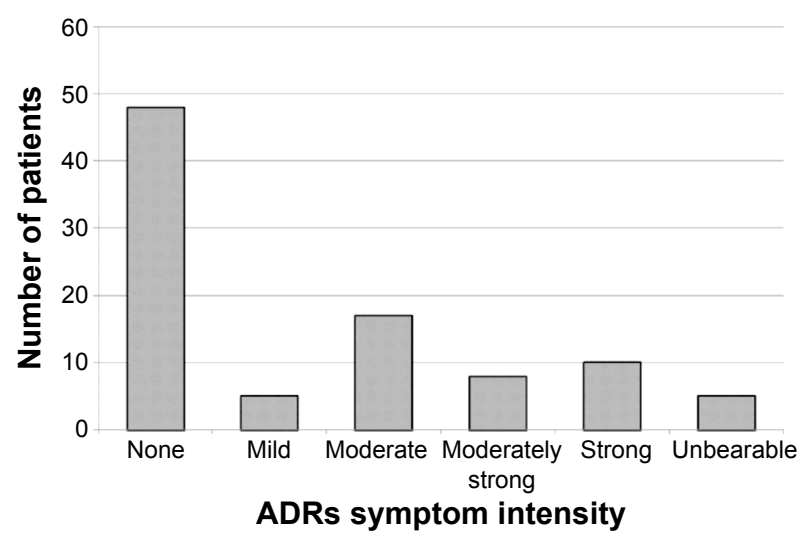

Figure 4 Distribution of adverse drug reactions (ADRs) reported according to the symptom intensity assessed by 5 -point visual analog scale (frequency histogram).

some questions remained unanswered (details about dealing with missing data are described in the "Methods" section). This result could indicate that patients would participate in the pharmacovigilance system if they had relevant information about the output (outcome of their participation). Also, it is very important to have a good and user-friendly reporting form, to work with a good coding system, and to have properly trained health workers in the pharmacovigilance system. The possible role of social networks in such a system has been extensively discussed elsewhere. ${ }^{9}$ However, patient reporting is not actively promoted in Serbia.

Eighty-two percent of participants were able to identify drug-related ADRs in their recent medical history. Rheumatology patients involved in our study are at high risk of ADRs and drug interactions due to several factors (eg, chronic diseases, multiple comorbidities, and co-medications). The combined treatment of chronic diseases and polypharmacy provide new challenges for health care cost control. ${ }^{16}$ Gäwert et al compared the information on ADRs obtained from patients with rheumatoid arthritis and their physicians. They confirmed the usefulness of patient reports but warned that serious ADRs might be omitted and the terminology might be different in comparison with health professional reports. ${ }^{17}$

Our survey was predominantly focused on possible ADRs experienced during the previous month. The time relationship between drug use and adverse event is one of the major parameters in the assessment of ADRs causality. If such a time lag is longer than a month, the assessment of causality will not be reliable, especially in patient reporting.

Surprisingly, a significant percent of patients from both groups were not aware of their diagnosis (group 1, 47.6\%; group 2, 68\%). According to Patient's Right Protection Law of the Republic of Serbia, patients should be informed on their diagnosis and treatment, including possible ADRs. ${ }^{11}$ However, our survey implies that communication between health workers and patients should be improved. Internet could be used as a valuable source of information for rheumatology patients. ${ }^{18,19}$ Internet resources could help rheumatology patients to overcome their decreased ability and to obtain the information needed. Of note, physicians tend to underestimate the proportion of their patients who obtain health information from the Internet. ${ }^{20}$

Marginal significance was found between rheumatology patients with and without neurological comorbidities (nervous system disorders) regarding their awareness of ADRs. Such a difference was not found in rheumatology patients with other comorbidities. According to Zhang et al, comorbidities significantly increase the risk of repeat admission for ADRs in elderly. ${ }^{21}$ However, nervous system disorders (cerebrovascular disease, dementia, and paraplegia) were comorbidities with a reduced likelihood of repeat hospital admissions compared to cardiology or rheumatology diseases. A repeat admission due to ADRs may contribute to patients' awareness of ADRs, but such a connection remains to be assessed. Little information is available on the prevalence of neurological disease in patients with a rheumatology diagnosis, but it is well known that neurological involvement in rheumatic diseases is associated with high morbidity and in some cases could be life-threatening. Serious neurological complications in rheumatic diseases appear to be rare. In addition, a lot of antirheumatic drugs may cause serious neurological ADRs. ${ }^{22}$

The majority of the patients who reported ADRs suffered from systemic diseases. It could be expected that the majority of patients in our survey reported ADRs of cytotoxic drugs. The most reported ADRs were moderate GUT discomforts. These data are different from the last few ALIMS annual reports on ADRs (data available at: http://www.alims.gov.rs/ eng/pharmacovigilance/). According to the ALIMS reports, the most frequent ADRs are cutaneous drug reactions, while GUT discomforts take the third place. Such a difference could be due to the safety profile of rheumatology drugs armamentarium. In addition, the sources of data are different in our survey and in ALIMS reports (patients and health professionals, respectively).

Finally, we may draw the profile of rheumatological patients willing to report ADRs: 1) the majority of them suffer from systemic inflammatory diseases and are more prone to neurological comorbidities; 2) they are predominantly aware of their diagnosis but less able to identify the drugs that may cause their ADRs; and 3) they reported mainly moderate gastrointestinal ADRs. Probably, the analysis of a larger 
sample may imply a different profile of patients willing to report ADRs, but it could not change the main conclusion of our study - that we should improve health education of rheumatology patients and their communication with health professionals.

We can suggest at least two strategies aimed at overcoming the reduced mobility of rheumatology patients and shortcomings of pharmacovigilance system observed in the present study: 1) strengthening the role of family physicians and other health workers in counseling and education of patients and 2) developing a network for online communication of patients with health care professionals. Both activities will improve patients' knowledge and attitude to drug safety.

Major weakness of our study might be the way of patient reporting of ADRs. Self-reporting of ADRs from the public is more reliable source of information than asking patients to report historic ADR at the time of admission to the hospital. However, our aims were to focus on the profile of rheumatology patients willing to report ADRs and to identify possible bias in such a reporting system. Conclusions from our survey might help health workers to overcome underreporting of ADRs and to establish firm network with their patients in pharmacovigilance.

\section{Conclusion}

This study confirms the important role of patients in system of pharmacovigilance. According to our knowledge, this is the first study that assesses the ADRs reported by rheumatology patients.

\section{Acknowledgments}

This study was supported by the Scientific Research Grant from Ministry of Science of Montenegro (No 01-1370) and by the Scientific Research Grant from the Ministry of Education and Science of Serbia (No OI 175046). This paper was proofread and edited by teaching assistant Danka Sinadinovic, Faculty of Medicine, University of Belgrade (Department of Humanities). We would like to thank Mrs Snežana Slavković for technical support during this study.

\section{Disclosure}

The authors report no conflicts of interest in this work.

\section{References}

1. Meyboom RHB, Lindquist M, Egberts ACG. An ABC of drug-related problems. Drug Saf. 2000;22(6):415-423.

2. Lazarou JBH, Pomeranz BH, Corey PN. Incidence of adverse drug reactions in hospitalized patients - a meta-analysis of prospective studies. JAMA. 1998;279:1200-1205.
3. Pirmohamed M, James S, Meakin S, et al. Adverse drug reactions as cause of admission to hospital: prospective analysis of 18820 patients. BMJ. 2004;329(7456):15-19.

4. US Department of Health and Human Services, FDA, Center for Drug Evaluation and Research Center for Biologics Evaluation and Research Center for Devices and Radiological Health. Guidance for industry patient-reported outcome measures: use in medical product development to support labeling claims; 2009. Available from: http://www. fda.gov/downloads/Drugs/.../Guidances/UCM193282.pdf. Accessed January 15, 2016.

5. European Medicines Agency. Fourth report on the progress of the interaction with patients' and consumers' organizations (2010) and results/ analysis of the degree of satisfaction of patients and consumers involved in EMA activities during 2010. EMA/632696/2011; 2011. Available from: http://www.ema.europa.eu/docs/en_GB/document_library/ Report/2011/10/WC500116866.pdf. Accessed September 15, 2015.

6. Law on medicines and medical devices adopted 2010 ("The Official Gazette of the Republic of Serbia", 30/2010). Available from: http:// www.alims.gov.rs/eng/regulations/law-on-medicines-and-medicaldevices/. Accessed January 3, 2015.

7. Rulebook on the method of reporting, collecting and monitoring adverse reactions to medicines ("Official Gazette of RS", No. 64/2011). Available from: http://www.alims.gov.rs/eng/files/2012/10/8-Rulee-onthe-method-of-reporting-collecting-and-monitoring-adverse-reactionsto-medicines_64-2011.pdf. Accessed January 3, 2015.

8. Mugoša S, Bukumirić Z, KovaČević A, Bošković A, Protić D, Todorović Z. Adverse drug reactions in hospitalized cardiac patients: characteristics and risk factors. Vojnosanit Pregl. 2015;72(11):975-981.

9. Edwards P. Questionnaires in clinical trials: guidelines for optimal design and administration. Trials. 2010;11:2. doi:10.1186/1745-6215-11-2.

10. Somers A, Petrovic M, Robays H, Bogaert M. Reporting adverse drug reactions on a geriatric ward: a pilot project. Eur J Clin Pharmacol. 2003;58:707-714

11. Higgins JPT, Green S, editors. Cochrane Handbook for Systematic Reviews of Interventions Version 5.1.0. The Cochrane Collaboration; 2011 [updated March 2011]. Available from: www.cochrane-handbook. org. Accessed December 8, 2015.

12. Blenkinsopp A, Wilkie $P$, Wang $M$, et al. Patient reporting of suspected adverse drug reactions: a review of published literature and international experience. Br J Clin Pharmacol. 2007;63(2):148-156.

13. Knezevic MZ, Bivolarevic IC, Peric TS, et al. Using Facebook to increase spontaneous reporting of adverse drug reactions. Drug Saf. 2011;34(4):351-352.

14. DeWitt JE, Sorofman BA. A model for understanding patient attribution of adverse drug reaction symptoms. Drug Inf J. 1999;33: 907-920.

15. van Hunsel F, Härmark L, Pal S, et al. Experiences with adverse drug reaction reporting by patients: an 11-country survey. Drug Saf. 2012; 35(1):45-60.

16. Clerc $\mathrm{P}$, Le Breton J, Mousquès J, et al. Drug-prescription management in patients with multiple chronic conditions. Results of the experimental polychrome study. Questions d'economie de la Sante. 2010;156:1-6. Available from: http://www.irdes.fr/espaceanglais/publications/ irdespublications/qes156.pdf. Accessed January 3, 2014.

17. Gäwert L, Hierse F, Zink A, et al. How well do patient reports reflect adverse drug reactions reported by rheumatologists? Agreement of physician- and patient-reported adverse events in patients with rheumatoid arthritis observed in the German biologics register. Rheumatology (Oxford). 2011;50:152-160.

18. Gordon MM, Capell HA, Madhok R. The use of the Internet as a resource for health information among patients attending a rheumatology clinic. Rheumatology (Oxford). 2002;41:1402-1405.

19. Schwartz KL, Roe T, Northrup J, et al. Family medicine patients' use of the Internet for health information: a MetroNet study. J Am Board Fam Med. 2006;19(1):39-45. 
20. Moretti FA, Oliveira VE, Silva EM. Access to health information on the internet: a public health issue? Rev Assoc Med Bras. 2012;58: $650-658$.

21. Zhang M, Holman CD, Price SD, et al. Comorbidity and repeat admission to hospital for adverse drug reactions in older adults: retrospective cohort study. BMJ. 2009;338:a2752.
22. Sofat N, Malik O, Higgens CS. Neurological involvement in patients with rheumatic disease. QJM. 2006;99(2):69-79.

\section{Publish your work in this journal}

Patient Preference and Adherence is an international, peer-reviewed, open access journal that focuses on the growing importance of patient preference and adherence throughout the therapeutic continuum. Patient satisfaction, acceptability, quality of life, compliance, persistence and their role in developing new therapeutic modalities and compounds to optimize clinical outcomes for existing disease states are major areas of interest for the journal. This journal has been accepted for indexing on PubMed Central. The manuscript management system is completely online and includes a very quick and fair peer-review system, which is all easy to use. Visit http://www. dovepress.com/testimonials.php to read real quotes from published authors.

Submit your manuscript here: http://www.dovepress.com/patient-preference-and-adherence-journal 